\title{
Computer Assisted Radiology: Symposium and Exhibition
}

\section{AIMS OF THE COMPUTER ASSISTED RADIOLOGY MEETINGS}

$\mathbf{T}$ HE FIRST Computer Assisted Radiology (CAR) symposium, CAR'85, was initiated with the aim to present and discuss the developments of computer and communication sciences in medical imaging. Aspects of these sciences, which were and still are of particular relevance include: digital image generation, image management and communication, medical work stations, application systems, and social aspects.

Since then, several CAR meetings (CAR'87, CAR'89) ${ }^{1,2}$ took place in Berlin as well as a joint meeting of CAR with the Society for Computer Applications in Radiology (SCAR), in June 1990, in Anaheim, CA. ${ }^{3}$

In summary, the last CAR meeting in Berlin, CAR'89, attracted 791 medical and scientific personnel from 27 countries. Altogether 190 separate contributions were the basis of tutorials, paper presentations, and workshops. It is interesting to note that 43 technical and medical journalists filed reports to the press, radio, and TV stations.

Visual demonstrations of developments in the field of computer assisted radiology and related activities were given in an industrial exhibition by 22 leading manufacturers of equipment, systems, and services.

The CAR'91 symposium is organized to introduce to the medical and technical community current achievements in digital imaging and computer assisted methods for diagnosis and therapy planning. It is realized in coordination with many international medical and technical societies, as well as educational institutions and industrial organisations.

CAR'91 is therefore intended to serve as a forum for discussion between experts from the medical sciences (eg, radiology, radiotherapy, nuclear medicine, orthopaedics, cardiology, neurology, and surgery) and professionals from the computer and physical sciences. The aim is to evaluate the sate of the art (presented in tutorials) and research results (session papers) in the area of computer assisted radiology.

For a successful symposium and exhibition, cooperation between representatives of the dis- ciplines concerned is essential. This is particularly relevant for support of national radiological societies and international organizations.

Activities of a cooperating society that are being considered to be in support of CAR are (1) to give recommendations, particularly to young radiologists, that CAR represents an important component in the future development of radiology; (2) to encourage research and development projects in the application areas of CAR; (3) to assist in assessing the relative strength and weakness of distributed radiological services in industrialised and nonindustrialised countries; (4) to assist in defining international standards for medical image processing, presentation, and communication; (5) to assist in evaluating the effects of new technologies on radiological training and to define further education programs; (6) to assist in defining the legal framework in which the new technologies should be operated; and (7) to delegate a representative of the cooperating society into the program committee, for example, for the compilation of the CAR symposium program, as well as the coordination with other meetings of the cooperating society.

Many European radiological societies, in addition to the American College of Radiology and the Radiological Society of Japan, have agreed to give their support. Several international societies with members from both the medical and technical communities also have a cooperation status with CAR'91.

EuroPACS and SCAR in particular document a wide international support by aligning their annual meetings with CAR'91. Also some specific European projects such as ISCAMI (Integrated Systems for Computer Assisted Management and Manipulation of Medical Images) ${ }^{4}$ and ECR-SCMI (European Committee on Recommendation for Standards of Computers in Medical Imaging) ${ }^{5}$ will be carrying out their workshop at the CAR'91 meeting.

Discussions are in progress for Eastern European Societies and projects to join CAR'91. All together, CAR'91 promises to be an exciting event with many new participants from various parts of the world. 


\section{PROGRAM}

In more detail, the CAR'91 program will contain the following topics for paper presentations:

Digital Image Generation: CT, MR, DSA, US, SPECT, PET, DR and Digital Fluoroscopy. Image Management and Communication (IMAC): Picture Archiving and Communication Systems (PACS), Digital Optical Recording and Communication, Local Area Networks, Distributed Radiology and Teleconferencing, ISDN, Multi-Media Data Base Management and Communication, PACS/Radiological Information System (RIS)-Interface, Standards, System Planning, Implementation and Evaluation.

Medical Work Stations: Computer Vision, Computer Graphics, Modelling and Human Computer Interaction.

Application Systems for: Computer Assisted Radiological Diagnosis, Computer Assisted Orthopaedics, Computer Assisted Radiation Therapy and Computer Assisted Surgical Planning.

Introduction to some of the above topics are provided through tutorials. They are presented in a dialogue fashion between an expert from the medical and computing field, respectively. Tutorials should give medical personnel concerned with digital imaging an appreciation of the advantages and pitfalls of computer and communication technology. For the computer professional in turn, they should provide a better understanding of clinical problems and goals related to medical imaging.

In addition, two further sessions on special topics have been scheduled for discussion. The first of these deals with the social aspects of CAR with all of its far-reaching prospects. Discussion themes are the effects of CAR on doctor and patient, the influence of CAR on medical training and expert systems as well as the social and economic influence of CAR on the health service. The future infrastructure of radiology will be dealt with here in particular.

The second special session is dedicated to a special group of patients: children. For the first time, within the framework of CAR, an attempt will be made to show how these new technological diagnostic procedures can support radiology for children, and how, when necessary, decisive support can be given for newborn children, infants, and adolescents.

The symposia will be accompanied by an extensive Industrial Exhibition displaying equipment, systems, and services. In the Scientific Exhibition-"work-in-progress"- research institutions present new CAR-related projects. A detailed Poster Exhibition with ample opportunities for discussion completes the range of projects on display

Heinz U. Lemke

Technical University

Berlin, Germany

\section{REFERENCES}

1. Lemke HU: Computer assisted radiology. J Digit Imag 2(1):9-10, 1989

2. Lemke HU: CAR'89-_Computer assisted radiology third international symposium and exhibition, Berlin, June 25-28, 1989. J Digit Imag 2(2):69-70, 1989

3. Bauman RA: S/CAR' 90 symposium on computer assisted radiology, Anaheim, CA, June 13-16, 1990. J Digit Imag 3(1):3-4, 1990
4. Lemke HU: Europeans begin to close PACS technology gap. Diag Imag:38-48, 1990

5. Lemke HU: Standard activities on IMAC in Europe. Proceedings of the First International Conference on Digital Radiology and PACS, Bologna, Italy, November 12-13, 1990 